\title{
SOCIAL MEDIA UTILISATION FOR COMMUNITY DEVELOPMENT: A STUDY OF NON PROFIT ORGANISATIONS IN MALAYSIA
}

\author{
Withaneachi $\mathrm{C}^{1^{*}}$ and Nagaraj $\mathrm{S}^{2}$ \\ ${ }^{1}$ INTI International University Nilai, Malaysia \\ ${ }^{2}$ Vels University, India
}

\begin{abstract}
With the increased legitimacy of social media in the eyes of key stakeholders, it has become more essential for non-profit organizations to adopt the use of information communication technologies for community development. The interactivity, user-friendly and cost-effective features have presented opportunities for NGO to communicate and engage with their public more effectively. In the case of Malaysia, the popularity of these social media applications with an adoption of $80 \%$ of online activities involving social media has heightened the need for the effective utilization of these tools. [mcmc.gov.my, 2016]. To gain a clearer understanding of the NGOs social media practices for community development, the researchers carried out an online ethnographic study of selected NGOs in the Klang Valley. In-depth interviews were also conducted to gauge their understanding and utilisation of these new communication technologies, as the study was exploratory in nature. The objective of the study was to examine the utilisation of social media practices of NGOs in Malaysia for community development. The findings demonstrated that social media practices of the NGOs were generally positive but despite having an impact on community development it was limited to being informational rather than engaging, hence limiting the potential of building and maintaining stronger relationships with the community.
\end{abstract}

Keywords: non-profit organisation [NGOs], community development, social media

\section{Introduction}

According to mcmc.gov.my, [2017], Internet users in Malaysia are made up of 24.5 million users who use the Internet as a source of information specifically, social networking [89.3\%] sites. With an average of four social media accounts ranging from Facebook to Instagram and an $80 \%$ adoption of social activities with an average time of four hours spent daily on them, social media has become a force to reckon with in Malaysia. [mcmc.gov.my, 2016]. Out of these four social media accounts, Facebook [97.3\% account ownership] reigns as the number 1 social media platform in Malaysia, with Instagram [56.3\% account ownership] in second place followed by Youtube [45.3\%] in the third spot. All these data demonstrate the increasing pervasiveness of the Internet and its social applications on everyday life, society and businesses.

To date, there has been an extensive number of research papers on the development of social media for businesses [Yusri, 2016], but social media research for organisation level research has not grown as extensively. Although there has been some organisational research on Facebook utilisation for nonprofits [Bortree \& Seltzer, 2009; Greenberg \& MacAulay, 2009; Waters et al., 2009]. Despite a mature Internet-savvy population, to date, there has been little no research on the usage and effects of social media utilisation in Malaysia. According to Sani (2017), e-commerce sales impact only made up of 1.1 $\%$ of total retail sales. This could point towards an untapped potential of the medium even for NGOs, 
as it is more cost-effective, easily accessible and flexible for the community. This research seeks to understand non-government organisation utilisation of these mediums to gauge whether the full untapped potential of these mediums have been utilised.

\section{NGOs and Social Media in Malaysia}

As the momentum of social media takes over Malaysia with $80 \%$ of users having ownership of various social media accounts with $90.1 \%$ of them using the Internet as a primary source of information [mcmc.gov.my, 2016] it is no surprise that business and non-government organisations have followed suit by creating their presence on these sites to capitalize on the potential of social media.

An impactful utilisation of social media saw a coalition of 62 non-government agencies under Bersih 2.0 utilised Facebook and Twitter to gain over 200,000 backers to ask the Prime Minister of Malaysia to quit, demonstrating the powerful role of social media in mobilizing change and development. [Fauziah Ahmad, et.al,2012]. Besides serving as a tool for political or democratic participation, social media has also been used in Malaysia to further other causes such as raising funds to mobilizing communities to join offline events such as \#PlankforMercy HumanitarianRun, Ikal Mayang Campaign and WOMEN's storytelling movement [The Star, 2015].

Social media presents a platform that allows for stronger attachments on already sound-foundations of specific causes, strengthens the sense of belonging and serves as a channel of encouragement to participate in civic and community issue that would not normally be accessible to most due to levels of exposure. The role of social media and its tool are fast becoming recognised as an important tool in community development. [Niven, 2011]. Realizing the strength of social media for community development, many human rights, educational, environmental and other types of NGOs have begun to participate in building their very own presence online via social media sites regardless of their size or cause.

\section{Background of the Study}

In the next section, the researcher will discuss the background of the study, the objectives, research questions and chosen methodology that will inform the study. Prior to the development of social media, NGOs were unable to utilise the many benefits of the Internet due to strategic or funding issues revolving around know-hows in creating a website, maintaining them and others. [Kent et.al, 2013; Saxton et al. 2007] thus failing to utilise them for stakeholder management or interactive communication rather merely informational purposes. The development of social media has allowed NGOs to explore increased possibilities for interpersonal engagement with a larger reach, interactivity of content and two-way dialogue with their respective communities for a limited cost.

According to Lovejoy and D.Saxton, (2012), social media carries a considerable potential as an organisational communication and stakeholder relations tool, this can be a motivating factor for Malaysian NGOs to join the bandwagon, like many other parties such as businesses, education and medical institutions. Hence, the importance of this study in understanding the utilisation of social media practices in Malaysia for community development. 


\section{Objective and Research Questions}

The objective of the study was to examine the utilisation of social media practices of NGOs in Malaysia for community development. The utilisation of social media practices of NGOs in Malaysia will be analysed further with the breakdown of the following research questions;

R1. How are non-government organizations using social media platforms in terms of functions?

R2. How do organizations vary in their reliance on social media platforms?

R3. How many social application platforms do they utilize and are aware of?

\section{Methodology}

Sampling of non-government organisations was done using a criterion sampling based on a predetermined criterion of importance. The pre-determined criteria involved the following conditions:

- Non-government organisations that have been established for a minimum of two years.

- Non-government organisations that have a minimum of one social media account.

- Non-government organisation that are involved in some form of community development regardless of areas of focus.

Upon fulfilling the pre-determined criteria, 5 organisations were chosen to become part of the sample;

1. Amnesty International Malaysia[AI Malaysia]

2. SUARAM

3. Women's Aid Organisation [WAO]

4. SOLS24/7

5. Foreign Spouses Support Group [FSSG]

As the researcher is interested in how people accomplish their identities, activities, settings and sense of order, an online ethnomethodological analysis will be employed for interviews. The intent of the research is to describe the social process experienced by non-government organisations that may have a generalizing effect. [Gulbrium and Holstein, 2002]. The sample targeted for the interviews have been chosen indiscriminately regardless of causes, size and popularity to represent or position themselves as social actors in that particular group using membership categorization to form the bigger picture of social media practices among NGOs in Malaysia.

In-depth interviews were conducted from November 2017 to January 2018 with the sample organisations via Skye calls to determine the utilisation of social media platforms in terms of content, reliance and numbers. During the beginning phases of analysis, the researcher employed the use of the information-community-action classification scheme [Lovejoy and D.Saxton, 2012] to analyse the raw material derived from the research data as identified in Table 1. 
Table 1 Classification of Social Media and Community Development

$\begin{array}{lll}\text { Classification } & \text { Major } & \text { Minor } \\ \text { Information } & \text { Utilisation } & \text { Recruitment } \\ & & \text { Announcement } \\ & \text { Organisation Content } \\ & \text { Events } \\ & & \text { Forwarded Content } \\ & \text { Feedback } & \text { Official Matters } \\ & & \text { Formality } \\ & \text { Customisation } \\ \text { Frequency } & \text { Urgency } \\ & & \text { Daily/Weekly/ } \\ & \text { Monthly/Ad Hoc } \\ \text { Eommunity } & \text { Engagement } & \text { Private Messaging } \\ & & \text { Wall Postings } \\ & & \text { Re-Tweets } \\ & \text { Shares/Imaging } \\ \text { Action } & \text { Localized /International } \\ & \text { Reach } & \text { Local/International Communities } \\ & \text { Volunteerism } & \text { On-the-ground, Online activism } \\ & & \text { Full-time, part-time, freelance, adhoc } \\ & \text { Employment } & \text { Active, Passive } \\ & \text { Contribution } & \end{array}$

To further supplement the research, the researcher become part of the community of online users of the various social media platforms used by the sample to record crucial information on social media positioning, frequency, the purpose of posts and levels of engagement. Utilising membership categorization, associated activities that belong to a set or device were used to identify the audience, in this case producers of the content. This study will provide researchers with a deeper understanding of social media practices of NGOs as online ethnographic research will allow for the exploration of how these producers use these platforms in their world and get an enriched sense of meaning through the analysis of communities online. It was apt to choose this method as the platforms analysed were social media platforms and provide a larger sense of familiarity. Through a collection of first-hand information via in-depth interviews, the researchers gained insight into the social media practices of these organisation and its level of contribution towards community development in Malaysia.

\section{Findings and Discussion}

Using the information-community-action classification scheme, the researcher will analyse the key findings of the data, as per Table 1, to answer all the objectives set out in the study. Insights from both data gained from both the interviews and online ethnographic data revealed interesting key findings in terms of utilisation of social media platforms specifically the Facebook platform.

NGOS were initially reached through their primary social media platforms via the private messaging function and later up with follow-up emails and phone calls prior to the interview. Researchers found 
difficulty in getting NGOs to respond promptly to request for interviews due to their busy schedules and lower level of priority in using social media tools for formal communication. The sample interviewed and studied have three to four social media platforms, namely Facebook, Instagram, Twitter and Youtube. All organisations used Facebook as the main medium to communicate with their audiences on social media. Other platforms like Youtube are used on an ad-hoc basic, depending on the availability of content.

While the other social media platforms were mostly used on an ad-hoc basis, depending on the availability of new content. This was especially true with the Youtube accounts of these nongovernment organisations. In the case of SOLS24/7, the content was on a project basis, with a frequency of 3 months once or sometimes a longer period, with videos created 2 years ago, as reflected below;

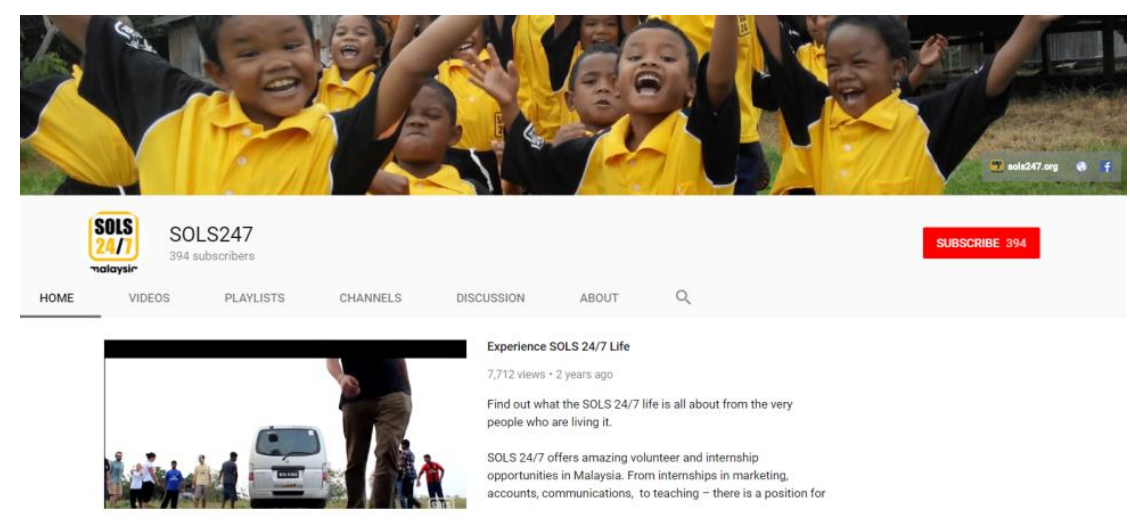

Figure 1: SOLS24/7 Youtube Channel

Despite the popularity of Twitter in other countries, Malaysia accounts for only $26.6 \%$ [mcmc.gov.my, 2017] that would reflect the lower usage of Twitter amongst NGOs in Malaysia. Most of the sample often use Twitter as a secondary tool for social media, for example SUARAM Malaysia creating automated content from Facebook for most of their postings with links;

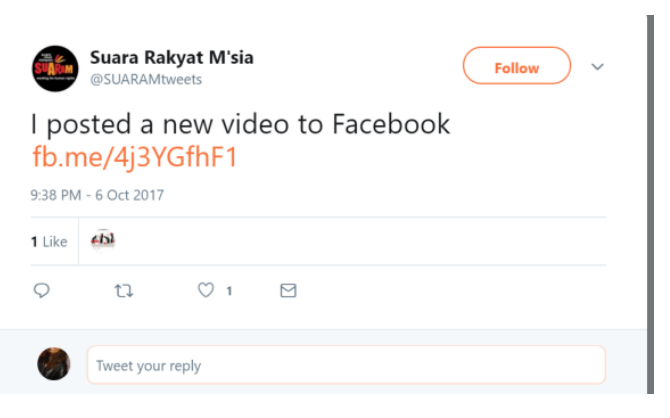

Figure 2: SUARAM Twitter Account

Besides that, it is also often used to re-tweet or forward content, mainly focusing on the informational aspect. AI Malaysia for example uses it to link to their global partners and organisations around the world while WAO used Twitter to run a poll to create engagement.

Instagram on the other hand was used as a lifestyle tool to follower influencers, tag location and provide solidarity likes to the other Amnesty branches. SOLS24/7 did use it more actively with Vlogs, tagging 
and even team introductions that helps build their brand. In the next session, the researcher will outline the findings according to each classification.

\section{Information Classification}

The information classification only works to inform rather than promote or mobilise others to take any form of action or two-way dialogue. This classification outlines the utilisation of content, feedback mechanisms and frequency of postings that can help the NGOs build credibility and networks. The researcher will also look into how this classification serves to work as a base to build on more advanced functions like dialogue.

One of the key observations of the online ethnographic study is that social media platforms of most of the organisations interviewed were used mainly for informational purposes rather than engagement. With Facebook, being the primary information exchange among all of these social media platforms, while the other social media platforms were mostly used on an ad-hoc basis, depending on the availability of new content. This was especially true with the Youtube accounts of these nongovernment organisations. Organisations that had Twitter as one of their accounts were either using it as a secondary tool through automated content from Facebook or only used it to re-tweet or forward content rather than for content creation.

In regards to the type of content that was utilised on these platforms, most of the organisations used Facebook to make announcements, post official matters and to send out information on event details. An interesting point to note is the FSSG group, which posts about 30 posts or more that focuses on immigration know-hows, job postings and any new updates to foreign spouses, often on a daily basis. Despite not having any event postings, FSSG group frequency of postings are very consistent in terms of employment opportunities, postings of articles, updates and posts by spouses. Other groups that use the Facebook platform to advertise employment opportunities are AI Malaysia, SOLS24/27 and WAO. SUARAM, WAO, SOLS24/7 focuses a lot on event information either sharing their own events or events relating to their causes.

Even though, Facebook reigned as the primary tool among these social media platforms, social media platforms were not seen as their primary communication channel to engage their audiences, instead, they still relied on mainstream media for audience reach as reflected by the following statement;

"Social media is great but there is a limitation, social media is a bubble, they agree with you already. It's like preaching to the choir. If you want to reach out to differently you have to go through different platforms. I find radio to be effective to reach out to people and to push forward". Heang Lee [WAO]

Although it was noted by SOLS4/27 that social media platforms like Facebook were crucial to reach cross-platform audiences, organisations like SUARAM used it primarily as an information platform to put all necessary communication on the organisation via Facebook. Among all the NGOs contacted, SUARAM, AI Malaysia, SOLS24/7 responded either almost immediately or within a few hours of messaging, indicating a higher reliance on Facebook as a medium for day-to-day operations. While WAO and FSSG used the automated message system indicating a pre-planned effort in responding to enquiries. 
Although when it came to follow-up of such messages, almost all of the respondents required a followup with email communications except for the FSSG that used Facebook as a primary tool. This demonstrated that social media platforms were still not considered as a primary tool for formal enquiries. Lack of consistent messaging and lower frequency of content via the Facebook messaging platform of the majority of these organisations was apparent especially among organisations that did not have a dedicated staff to handle their social media platforms exclusively.

In these platforms, access was held by three to four people, hence the inconsistency in messaging and content creation. This situation however was not experienced by organisations like AI Malaysia, FSSG and WAO that both had dedicated person to manage these platforms. Although it is interesting to note that the FSSG is run solely on a voluntary basis with no paid staff.

Through collection of online ethnographic field notes, the researcher noticed that content creation among the two organisation AI Malaysia and WAO was also more consistent in terms of frequency and type of content was more personalised. With the type of content ranging from announcements, use of infographics and quotes as reflected below in Figure 3 and Figure 4 ;

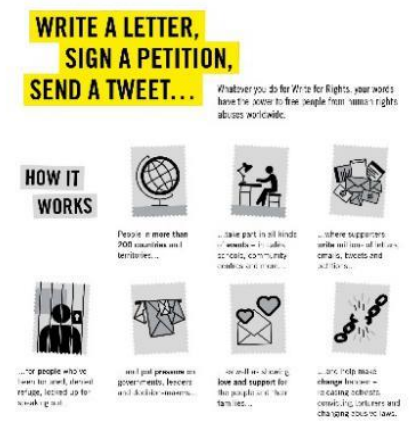

Figure 3: Al Malaysia's Action Post

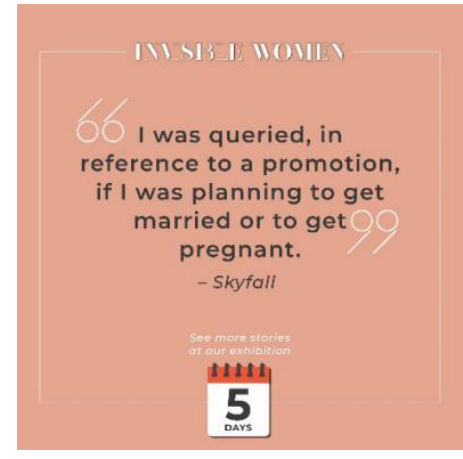

Figure 4: WAO's Invisible Women Campaign

Another point to note is that most of these organisations did not provide a link from their main social media platforms to other platforms, to encourage users to like, share or view those sites, signaling a loss of opportunity to provide crucial information that could boost the organisation and allow their members to engage. In the next section, we will analyse the second classification which is community to gauge if the sample organisations have gone beyond sending out information to creating dialogue among the members of their online community.

\section{Community Classification}

The community classification works to gauge if these organisations are creating dialogue or interacting with the members of their groups and engaging others to do the same. This function looks at how organisations not only place information but the mechanisms that are in place to facilitate interactions through sharing and online conversations that will in turn create an online community of followers.

To comprehend this classification scheme, two main aspects analysed are social media posts that spark conversations with their publics within their respective pages and the other is the strengthening of ties to the community without the necessity or expectation of interactive conversation. This is crucial as 
current studies on social media show an existing gap that exists between information and the creation of dialog. (Waters, et al. 2009).

After analysing online ethnographic data that was further supplemented with interviews with the sample, the community classification is outlined by engagement, language and reach to gauge the level of dialogue and community building that is present within this context. In terms of engagement, most organisations that were sampled did not have many responses to their respective posts on their social media, if engagement did exist it was very minimal and ad-hoc depending on the type of posting. Heang Lee, WAO communication officer, commented:

"Our engagement is not great, because we do we don't have the time, because it takes a lot of time. Mostly we have only likes and shares, more than actual comments, because our engagement is not strong. We do get private messages daily on students wanting to do interviews, people wanting to do project, to access our services, general enquiries and advice on gender based violence. So I try to look at it daily, but at times it slips my mind."

Echoing a similar tone, AI Malaysia's communication officer, Nisshanthan Dhanapalan said that capacity was a main issue, further commenting on the engagement aspect;

"People don't really comment. Lot of likes, we do have usual suspects, we do have regulars, 2-3 people who tend to send in positively cryptic messages that we do not know how to respond to, we don't respond to comments rather only message. We get about 56 private messages daily but we don't respond as fast, as we would love to do it a corporate level but capacity is an issue. We try to get back to them in a couple of days."

Despite a lower number of likes/followers, as compared to the other organisations [as reflected in the Table 2], FSSG's engagement in regards to responses to wall posts and page reviews were considerably high, with comments and replies on almost all posts, coupled with likes and shares. Some posts saw comments ranging from 5 to 47 comments depending on the issue posted. Responses to members were often swift from the administrators of the group and also came from the members themselves creating a robust and vibrant online community.

Table 2 : Numbers of Followers/Members of Facebook

\begin{tabular}{lcc}
\hline Social Media & Organisation & Likes/Followers \\
\hline Facebook & SOLS24/7 & 18,837 \\
& SUARAM & 100,364 \\
& WAO & 18,631 \\
& FSSG & 5,429 \\
& AI Malaysia & 30,606 \\
Twitter & & \\
& SOLS24/7 & 3,292 \\
& SUARAM & 13,300 \\
& WAO & 4,570 \\
& FSSG & n/a \\
& AI Malaysia & 2,739 \\
& & 755 \\
& SOLS24/7 & 412 \\
& SUARAM & 1,542 \\
\hline
\end{tabular}




\begin{tabular}{lcc}
\hline & FSSG & n/a \\
AI Malaysia & 945 \\
& & \\
Youtube & SOLS24/7 & n/a \\
& SUARAM & 128 \\
WAO & $\mathrm{n} / \mathrm{a}$ \\
FSSG & 87 \\
& AI Malaysia &
\end{tabular}

*Youtube terms followers as subscribers

While the FSSG, most often featured content of its members asking questions to the audience and documents that provided guidelines to spouses. In terms of reaching out to a bigger audience, AI and SOLS24/7 did have a larger international reach as compared to the other organisations that were mostly localised, due to their affiliations with other countries through their other branches in countries like Australia, Netherlands, Indonesia and others.

Contributors on the various platforms were deemed more passive although it was noted that they were cheerleaders for each organisation that was regulars who would like and comment on every post and were often from the same fields creating somewhat of an echo chamber. Many of the organisations like SUARAM, WAO, FSSG attributed the lack of engagement due to the strategy and implementation of desired outcomes on their platforms. Dobby Chew of Suaram further noted;

"We lack implementation of the strategy. We have a huge following, but little engagement. No strategy. People don't know how to use Facebook, the general belief is that anything that you post, everyone will see."

But organisations like SOLS24/7 noted that seasons or timing was crucial towards engagements remarking that it can be seasonal, depending on time and batches. Conversations online with their respective communities were noted to be an important tool to gauge how these organisations engaged their audience. It is noticed that most organisations did have ultimate or defining conversations as reflected below;

"We once engaged with our audience when the word Jakun was used, it was an excellent time to reconnect with audiences." - Danutcha, [SOLS 24/7]

"Detention Without Trial case, we used 10 bullet point featuring case date, who was involved, why they were arrested. It got us 1700 shares. It blew up and went all over the place"- Dobby Chew [SUARAM]

\section{Action Classification}

In this section, we will analyse the third classification which is action to gauge if the sample organisations are able to get their followers, subscribers and other community members to "take an action" for the organisation. "Take an action" could range from giving a donation in terms of cash or kind, providing expertise, buying merchandise of the organisation, attending events, volunteering and engaging themselves in advocacy campaigns and becoming activist themselves. The primary function of the action classification in this classification is to demonstrate if this organisation's social media messages are able to convert their members to help the organisation in its mission. To comprehend this 
classification scheme, three main aspects that will be analysed based on the interviews and social media postings from the various platforms are volunteerism, employment and contributions. This section is extremely important as it will outline whether the sample has been able to utilise their social media platforms to mobilise their members.

SOLS247 for example received a lot of enquiries online from different countries that often turned into on-the-ground volunteers from various countries. While Amnesty had various campaigns that encouraged people to take action through their letter-take action. It is interesting to note that in terms of employment, the current communication officer of AI Malaysia was hired full-time via the Facebook platform even though it wasn't on the organisation platform but the bosses' personal account, shows the enormous potential of this platform for mobilisation.

WAO often uses the Facebook platform to promote their events that often gets quite a bit of traction in terms of attendance sign-up totaling to about 250 people but at the actual event only about 70 people turn up. So although the numbers do not reflect accurately, WAO feels that it does create an awareness of the event to some parts of the society. On the other hand, AI Malaysia remarked that it was hard to quantify the level of action from the side of their audiences via Facebook for instance. According to Nisshanthan;

"We get volunteers via social media, but there is a group of silent audience members, they won't say a thing, they do see our post, but there is no way of quantifying that."

None of the organisations used the platform to receive donations in terms of cash or kind effectively, but FSSG for example used it to gain expertise from community members to advice on issues, provide opportunities and linkages.

It was evident that non-government organisations used social media applications mainly for informational purposes due to the lack of resources to place more emphasis and importance on engagements and insights of the use of such applications although there is some impact on community development. A majority of them who were managing these applications were not trained in this field and were in-charge of multiple roles within the organisation, often limiting the potential to further grow their online audience through community and action. The next section will discuss the conclusion of the study and ways to move forward to leverage on social media platforms.

\section{Conclusion}

A review of the findings of this study points to a deeper insight into the workings of non-government organization in the realm of social media applications for information, community and action. Despite the expected potential of these platforms for usage, findings reflect that despite a higher potential to utilise these platforms for community and action, these organisations fall short in leveraging on the potential of these platforms due to manpower and financial constraints. This truly points to a more complicated relationship between non-government organisations and social media. Even though there is a low entry cost in using these platforms as an important communication tool, most organisations are still holding a very traditional view of media, not putting much emphasis or placing much reliance on this form as a major medium.

Although the subjects that were interviewed realized the importance of placing more emphasis on these platforms, they still lacked the level of expertise due to the limited experience in running social media, 
their background and the lack of manpower. More importantly, the study points to the necessity of putting more emphasis on understanding the economical limitation of non-government organisations as compared to business entities. A utopian view of social media platforms cannot be used to apply to all organisations due to the different social-economic backgrounds of these organisations.

These platforms have a high potential for the development of awareness in the various social causes advocated by these NGO especially to the younger generation in Malaysia. But key findings points to the utilisation of it primarily for information rather than community or action, although it would be unfair to not acknowledge that some measures were taken to utilise the community and action classification that has contributed to a lower level impact on community development.

There are some major topics that emerged to inform this study namely the social-economic situation of the non-government organisation, training and expertise and exposure to the use of such mediums. Although the results of this study are still considered preliminary, it is evident that non-government organisations, are caught in a double-edge sword situation, whereby they are aware of the importance of these platforms but are put off by the lack of resources to maximise the potential of these mediums for community development. It is hoped that a future study will explore how NGOs can leverage on existing resources to utilise this untapped platform to their advantage.

\section{Acknowledgments}

This research paper was made possible with the support of non-government organisations in Malaysia namely SUARAM, AI Malaysia, WAO, FSSG and SOLS24/7 who agreed to become part of the interview sample. Not to mention, North South Initiative's Executive Director, Mr. Adrian Pereira for his assistance in linking up the research to potential interviewees.

\section{References}

Bortree, D. and Seltzer, T. (2009). Dialogic strategies and outcomes: An analysis of environmental advocacy groups' Facebook profiles. Public Relations Review, 35(3), pp.317-319.

Greenberg, J. and MacAulay, M. (2009). NPO 2.0? Exploring the Web Presence of Environmental Nonprofit Organizations in Canada. Global Media Journal: Canadian Edition, Volume 2, Issue 1, pp. 63-88.

Gubrium, J. and Holstein, J. (2004). Handbook of interview research. Thousand Oaks, Calif: Sage Publications.

Lovejoy, K. and Saxton, G. (2012). Information, Community, and Action: How Nonprofit Organizations Use Social Media*. Journal of Computer-Mediated Communication, 17(3), pp.337-353.

Fauziah Ahmad, Chang Peng Kee, Normah Mustaffa, Faridah Ibrahim, Wan Amizah Wan Mahmud \& Dafrizal. (2012). Information propagation and the forces of social media in Malaysia. Asian Social Sciences Journal, 8, 71-76.

Kent, M. L., Taylor, M., \& White, W. J. (2003). The relationship between Web site design and organizational responsiveness to stakeholders. Public Relations Review, 29, 63-77.

Mcmc.gov.my. (2016). [online] Available at: https://www.mcmc.gov.my/skmmgovmy/media/General/pdf/IUS2016.pdf [Accessed 16 Nov. 2017]. 
Mcmc.gov.my.

(2017).

[online]

Available

at:

https://www.mcmc.gov.my/skmmgovmy/media/General/pdf/IUS2017.pdf [Accessed 15 Jan. 2018].

Niven, Rosie (2011, December, 8). The Role of Social Media in Community Development. The Guardian. Retrieved on 5 January 2018, https://www.theguardian.com/voluntary-sectornetwork/community-action-blog/2011/dec/08/facebook-social-media-community-development

Sani, Rozana (2007, March 22). Marketing in Digital Spaces. News Straits Time. Retrieved from https://www.nst.com.my/news/2017/03/223452/marketing-digital-space

Saxton, G. and Guo, C. (2009). Accountability Online: Understanding the Web-Based Accountability Practices of Nonprofit Organizations. Nonprofit and Voluntary Sector Quarterly, 40(2), pp.270-295.

The Star (2015, August 31). Connecting through social media. Metro Focus. Retrieved on 2 January 2018, https://www.thestar.com.my/metro/focus/2015/08/31/connecting-through-social-media/

Waters, R., Burnett, E., Lamm, A. and Lucas, J. (2009). Engaging stakeholders through social networking: How nonprofit organizations are using Facebook. Public Relations Review, 35(2), pp.102106.

Yusri, Muhammad Ihsan (2016) Investigation On Social Media Adoption Among The Food Truck Vendors In Malaysia. Project Report. UTeM, Melaka, Malaysia. (Submitted) 